\title{
Sociological Impact of COVID-19 on People with Non-Communicable Diseases (NCD) and Long COVID-19 in Young Children
}

\author{
Kamoka Hana Moftah Ibrahem ${ }^{1}$, Asita Elengoe ${ }^{1 *}$, Abdulmutalib Alabeed Allaq², Alabed Ali \\ Ahmed Alabed ${ }^{3}$ \\ ${ }^{1}$ Department of Biotechnology, Faculty of Science, Lincoln University College, 47301 Petaling Jaya, Malaysia \\ ${ }^{2}$ Faculty of Applied Sciences, Universiti Teknologi MARA, 40450 Shah Alam, Selangor Darul Ehsan, Malaysia \\ ${ }^{3}$ Department of Community Medicine, Faculty of Medicine, Lincoln University College, 47301 Petaling Jaya, \\ Malaysia
}

Article history:

Submission July 2021

Revised July 2021

Accepted September 2021

*Corresponding author:

E-mail: asitaelengoe@yahoo.com

\begin{abstract}
The Coronavirus Disease 2019 (COVID-19) pandemic has posed enormous health risks worldwide. In the $21^{\text {st }}$ century, a novel virus which is called SARS-CoV-2; was the main cause for the COVID-19 pandemic outbreak. This virus is transmitted from person to person through direct and indirect transmission. Some infected people develop serious symptoms, but others do not show any symptoms. Infected people with no visible symptoms could also transmit the viruses. Literature studies about the COVID-19 pandemic (starts from Dec 2020 until June 2021) were conducted through PubMed, Embase, Medline and Google Scholar. In this study, we explored the COVID-19's impact on non-communicable diseases and young children. Scientists are currently testing several drugs to eradicate the COVID-19 outbreak. Pfizer, AstraZeneca, Sinovac and Moderna, vaccine doses administrated successfully throughout the world. Government agencies also implemented certain rules and regulations to protect their citizens from coronavirus. Moreover, World Health Organization (WHO) also helps by providing many valuable information and precautions to countries. Hence, this study might provide some information on how to deal with the virus to curb the rapid spread of the SARS-CoV-2.
\end{abstract}

Keywords: COVID-19, Non-communicable diseases, SARS-CoV-2, Young children

\section{Introduction}

In 2003, severe acute respiratory syndrome (SARS) became a serious health threat to the public. It is a zoonotic respiratory disease caused by SARS coronavirus (SARS-CoV). It was responsible for the epidemic outbreak in Guangdong, China. Research studies reported that the virus transmitted from bat to human [1]. In December 2019, a novel coronavirus strain was first discovered in Wuhan, China. It was the SARS-CoV-2. It is highly pathogenic compared to the SARS-CoV strain [2]. In January 2020, the World Health Organization (WHO) designated the outbreak as a Public Health Emergency of International Concern and named it COVID-19. They declared the outbreak as a pandemic in March 2020. As of
August $25^{\text {th }}$, 2021, COVID-19 had been linked to almost 214 million illnesses and 4.47 million deaths, making it one of the deadliest pandemics in history [2].

Currently, there are several variants identified from the mutation of the COVID-19 strain. These variants are namely P.1, N501Y, 501Y.V2 [3] and B.1.617.2 (delta) variant [4]. Among these variants, delta variant poses a serious threat such as a fatal acute respiratory system which cause a hike number of cases in India [4] and P.1 originated from Brazil, which has less concern, whereas N501Yoriginated from the United Kingdom with increased virulence while 501Y.V2 is a variant from South Africa linked to greater viral load thus

\section{How to cite:}

Ibrahem KHM, Elengoe A, Allaq AA, Alabed AAA (2022) Sociological Impact of COVID-19 on People with Non-Communicable Diseases (NCD) and Long COVID-19 in Young Children. Journal of Tropical Life Science 12 (1): 63 - 71. doi: 10.11594/jtls.12.01.06. 
worst fatality [3]. These variants differ from the original Wuhan variant, which influences the function of spike glycoprotein as the alteration of the spike glycoproteins affects the interaction with the human angiotensin-converting enzyme 2 (hACE 2) receptor [5].

SARS-CoV-2 targets the lung as the primary organ. However, clinical studies revealed that various organs such as the heart, blood arteries, kidneys, gut and brain are also infected by the virus. COVID-19 symptoms are highly diverse, ranging from none to life-threatening sickness [6]. COVID-19 affects older people, especially 70 years old above; people with chronic diseases like respiratory-related diseases, diabetes, hypertension, kidney-related diseases; and immunocompromised people (e.g. cancer and HIV patients) badly. COVID-19 is an airborne transmission disease. SARS-CoV-2 spreads very fast from human to human via air when they are close together. SARS-CoV-2 spreads from one infected person to another person through lips, nose, or eyes as people breathe, cough, sneeze, or speak. Therefore, SARS-CoV-2 also transmits via water droplets. SARS-CoV-2 is also feasible that it will spread via infected surfaces. Asymptomatic carriers can transmit the virus to a healthy person. They can spread it for up to 2 weeks. An asymptomatic carrier does not show any signs and symptoms of COVID-19 but the virus within him or her for 2 weeks [6].

The virus transmission can be prevented through strict operating procedures such as maintain a social distance of about 1 meter; wear a face mask whenever outside the house; wash hands frequently with a sanitizer, soap and water; and selfisolate if a person is infected with a virus or had close contact with an infected person. All the important places like supermarkets, grocery shops and restaurants should always sanitize the surfaces such as trolleys, refrigerator doorknobs, tables, chairs, etc. Proper ventilation and air filtration can curb the spread of the virus. Home quarantine is frequently used to assure safety, either as a result of travel or after exposure to an infectious individual, as advised by the WHO [6]. This home quarantine is based on the disease's incubation period and its infectivity. Vaccines are being created and distributed in various ways to reduce the spread of the virus. While work is undone, current treatments focus on alleviating symptoms.

Since the SARS-CoV-2 virus and its genome were discovered, the scientific community has put forth an extraordinary effort to develop an antiviral drug or vaccine. This effort has resulted in the development of over 300 vaccine proposals [7]. According to World Health Organization (WHO) vaccine tracker and landscape, 112 vaccines were evaluated in clinical development and 184 in preclinical development [8]. A few of these novel vaccines have received emergency approval. Existing evidence suggests that new vaccine candidates could help protect people and slow the development of a pandemic. Because the conceptual and technological platforms used are so diverse, certain vaccinations are likely to be better suited to different segments of the human population. Furthermore, it is uncertain if and to what extent the vaccines in question, as well as other vaccines such as BCG, might increase immunological fitness by training innate immunity to SARS-CoV-2 and pathogen agnostic protection [9].

The COVID-19 pandemic is threatening the world's economic and health systems, highlighting the degree of global interdependencies and the importance of global health hazard preparation. To date, on $25^{\text {th }}$ August 2021, a total of 213,999,186 cases of COVID-19; 4,465,417 death cases and 191,485,881 recovery cases have been reported worldwide [10]. Pfizer, AstraZeneca, Sinovac and Moderna, vaccine doses administrated successfully throughout the world. This study reviewed the sociological impact of COVID-19 on people with non-communicable diseases (NCD) and long COVID-19 in young children. The world is still in search of more solutions to the COVID-19. Therefore, this study might provide some information on how to deal with the virus in terms of preventive measures and control the rapid spread of the SARS-CoV-2 virus in the future.

\section{COVID-19's impact on non-communicable dis- eases (NCD)}

COVID-19 infection and mortality are more frequent in those with chronic lung, kidney and liver diseases, diabetes mellitus (DM) and cancer patients. Table 1 describes the prevalence percentage of comorbidities among COVID-19 patients.

\section{Pulmonology}

SARS-CoV-2 infection and mortality are exacerbated by chronic respiratory illnesses, which are among the most potentiate of SARS-CoV-2 infection and mortality [27]. Cigarette smoking has 
Table 1. Prevalence percentage of comorbidities in COVID-19 patients

\begin{tabular}{|c|c|c|c|c|c|}
\hline Author & Year & Disease & $\begin{array}{l}\text { Sample } \\
\text { size }\end{array}$ & $\begin{array}{l}\text { Prevalence percentage of } \\
\text { comorbidities (\%) }\end{array}$ & Reference \\
\hline Onder et al. & 2020 & Diabetes & 355 & 35.5 & {$[11]$} \\
\hline Zhang et al. & 2020 & Diabetes & 140 & 12.1 & {$[12]$} \\
\hline Huang et al. & 2020 & Diabetes & 41 & 19.5 & [13] \\
\hline Wang et al. & 2020 & $\begin{array}{l}\text { Chronic kidney dis- } \\
\text { ease }\end{array}$ & 138 & 2.9 & {$[14]$} \\
\hline Bhatraju et al. & 2020 & $\begin{array}{l}\text { Chronic kidney dis- } \\
\text { ease }\end{array}$ & 24 & 21 & [15] \\
\hline Guo et al. & 2020 & $\begin{array}{l}\text { Chronic kidney dis- } \\
\text { ease }\end{array}$ & 187 & 3.2 & {$[16]$} \\
\hline Guan et al. & 2020 & $\begin{array}{l}\text { Chronic obstructive } \\
\text { pulmonary disease }\end{array}$ & 1099 & 10.5 & [17] \\
\hline Lian et al. & 2020 & $\begin{array}{l}\text { Chronic obstructive } \\
\text { pulmonary disease }\end{array}$ & 788 & 10.9 & [18] \\
\hline Guan et al. & 2020 & $\begin{array}{l}\text { Chronic obstructive } \\
\text { pulmonary disease }\end{array}$ & 1590 & 10.5 & [19] \\
\hline Liang et al. & 2020 & Lung cancer & 1590 & 18.5 & [20] \\
\hline Xun Li et al. & 2020 & Lung cancer & 25 & 18.2 & [21] \\
\hline Zhang et al. & 2020 & Lung cancer & 28 & 17.3 & {$[22]$} \\
\hline Rentsch et al. & 2020 & Chronic liver disease & 3789 & 15.4 & [23] \\
\hline Praveen et al. & 2020 & Chronic liver disease & 49 & 24 & [24] \\
\hline Phipps et al. & 2020 & Chronic liver disease & 2273 & 6.4 & [25] \\
\hline Reyes-Bueno et al. & 2020 & Dementia & 23 & 43.4 & [26] \\
\hline
\end{tabular}

been reported to be an independent risk factor for COVID-19 susceptibility in several studies, with males having a higher case fatality rate than females, owing to males' higher smoking rates [28]. This finding could also apply to e-cigarettes and other vaping devices [29]. In addition, the physical act of swapping cigarettes and hookahs, which is popular in many cultures, could provide SARSCoV-2 infection [30].

Bronchoscopy (used for confirmation diagnosis in suspected COVID-19 patients with negative nasopharyngeal tests) is a high-risk method for operators due to the proximity to the airway. Early elective intubation and ventilation are required to protect healthcare workers [31]. Patients with COVID-19-related critical respiratory failure and patients with significant exacerbations of chronic lung disease without COVID-19 infection have also competed for mechanical ventilators. Early elective intubation and ventilation are essential to safeguard healthcare workers since SARS-CoV-2 is easily aerosolized by high-flow nasal cannulas, bag valve masks, and non-invasive positive-pressure breathing (e.g. Bilevel Positive Airway Pressure) [32]. Although most patients who survive acute COVID-19 infection recover completely clinically, there have been cases of long-term lung injury, referred to as a spots COVID-19 fibrosis
[33]. This prospective illness could result in irreversible pulmonary deterioration in people who already have chronic lung disease, leading to the emergence of additional patients with interstitial lung disease.

According to the data from the Ministry of Health and Welfare and the Health Insurance Review and Assessment Service in Korea, a statewide retrospective cohort research was carried out with 4610 COVID-19 positive patients aged 40 years. The study was performed between $20^{\text {th }}$ January 2020 and $27^{\text {th }}$ May 2020. A multivariate logistic regression model was used to examine the clinical course and numerous clinical variables of chronic obstructive pulmonary disease (COPD) and non-COPD patients and the risks of respiratory failure and all-cause death in COPD patients. COPD patients received a higher proportion of mechanical ventilation and intense critical care among COVID-19 patients. In Korean COVID-19 patients, COPD is an independent risk factor for all-cause mortality [34].

According to Semra Bilaçeroğlu's (2021) study, it has been demonstrated that the numbers of bronchoscopic and non-bronchoscopic procedures were declined by 48.0 percent and 21.9 percent, respectively, during the pandemic [35]. As an aerosol-producing operation, bronchoscopy 
poses a challenge for healthcare providers during the epidemic. In addition to adopting aerosol reduction and viral transmission-reducing methods, a tier system for prioritizing the urgency of bronchoscopic operations and postponing elective ones is a sensible way for decreasing the potential transmission of SARS-CoV-2. In Turkey, similar tier systems and protocols have helped to reduce the number of conventional and interventional bronchoscopic treatments. A less stringent tier system was used because non-bronchoscopic (pleural and transthoracic) surge-ries are not high-risk aerosolgenerating procedures.

\section{Nephrology}

In the United States, the requirement for present or impending hemodialysis (HD) increases the risk of COVID-19 exposure for approximately 750000 end-stage renal diseases (ESRD) patients [36]. Because HD patients require frequent dialysis, long-term self-isolation is not an option. Furthermore, many persons with ESRD patients rely on others to transport them to and from dialysis centers, exposing them to virus carriers in the community [37]. Chronic kidney disease (CKD) is an immunosuppressive illness that causes infection resistance to decrease [38]. Many CKD patients also take ACE inhibitors and angiotensin-receptor blockers (ARBs), which could make them more susceptible to SARS-CoV2 [39]. The US CDC recommends repositioning dialysis chairs to comply with minimum six-foot social distance requirements [40]. Although some hospitals are now providing masks to patients, screening them for COVID-19 symptoms at check-in, and sending them away if they are positive, this drives patients to emergency departments, where they are more likely to contract the illness if they were previously negative [41]. Only a small part of patients (roughly 12, 000) undergo peritoneal dialysis (PD). Those who want to change from HD to PD will need surgical dialysis catheter placement, which is not specifically feasible at present due to a lack of surgical capacity, anesthesia, and critical care services [42].

Based on ERA-EDTA Council, ERACODA Working Group study, it has been reported that dialysis (adjusted hazard ratio (aHR): 3.69), organ transplantation (aHR: 3.52) and CKD patients (aHR: 2.52) were the highest mortality risk for COVID-19 infection [43]. According to research performed by Dialysis Clinic Inc., a countrywide not-for-profit dialysis provider with 260 outpatient dialysis clinics catering for more than 15,000 maintenance dialysis patients, dialysis patients are at high risk of acquiring COVID-19 [44].

\section{Hematology and Oncology}

COVID-19 has a higher chance of causing unfavorable effects in those with hemoglobinopathies such as sickle cell anemia and thalassemia $[30,41,45]$. Patients undergoing chemotherapy or bone marrow transplants are also immunocompromised because of the myelosuppressive effects of several of these drugs. Cancer survivors, as well as patients with persistent disease, are more likely to die or require mechanical ventilation as a result of SARS-CoV-2, according to the Chinese COVID-19 experience [41, 46] due to the myelosuppressive effects of many therapies. On the other hand, chemotherapy or immunosuppressive medications may cause a diminished immune response, lessening the risk of cytokine storm, a COVID-19 complication that is feared [47]. Furthermore, SARS-CoV-2 has been linked to a coagulopathy condition that manifests as thrombosis in various arterial sites, including the brain, lung, and heart. COVID-19 also provides many significant systemic issues for oncologists and cancer patients. Cancer screenings are not without risk and can cause diagnoses to be delayed. Provider reassignment, social distance needs, and limited operating room capacity are all factors to consider [48]. During epidemics, chemotherapy, radiation treatment and surgical removal of malignancies may all be postponed. The majority of chemotherapy is administered in infusion centers, redesigned to maintain six-foot social separation requirements [49].

Based on Remuzzi and Remuzzi's (2020) study, it has been demonstrated that COVID-19 was responsible for 19.4 percent of fatalities among cancer patients [50]. The development of poor clinical outcomes in SARS-CoV-2 infections appears to be strongly linked to chemotherapy treatment. COVID-19 is thought to be highly risky for cancer patients.

According to Liang et al.'s (2020) research, individuals having systemic treatment (chemotherapy or immunotherapy) or surgery had a higher incidence of significant COVID-19 events, which increased even more in older patients when compared to patients without cancer [51]. 


\section{Otolaryngology, Gastroenterology and Hepa- tology}

COVID-19 patients presenting for medical care in some studies reported gastrointestinal symptoms such as diarrhea, vomiting, and abdominal pain [41, 52]. Operators are at risk of SARS-CoV-2 exposure during procedures such as esophagogastroduodenoscopy, colonoscopy and ear, nose, and throat operations [53]. According to Kumar et al. study (2003), it has been demonstrated that endoscopy suites have been turned into overflow intensive care unit (ICU) beds at several facilities, endoscopy procedures have been systematically limited since 2003 [54]. Several cases with inflammatory bowel disease (IBD) use immunosuppressive medicines such as corticosteroids and biologics, putting them at risk. Some experts recommend converting IBD patients on infusion anti-TNF biologics (infliximab) to home injectable formulations such as adalimumab to reduce SARS-CoV-2 exposure in infusion centers. Chronic liver disease (CLD) patients may also have a greater chance of poor COVID-19 results $[45,32]$. Based on previous new coronavirus outbreaks, liver transplants, in particular, provide a mechanism of viral transmission [55].

According to Wang et al. (2020) study, it has been reported that COVID-19 patients with a recent encounter for CLD had a mortality rate of $10.3 \%$ when compared to COVID-19 patients without CLD who had a death rate of 5.5\%. Moreover, the hospitalization rate for COVID-19 with CLD was 41.0 percent but COVID-19 without CLD had 23.9 percent. Both the mortality and hospitalization rates were significantly $(\mathrm{p}<0.001)$ proven in Wang et al.'s (2020) study [56].

A retrospective analysis found that 39 of 202 confirmed COVID-19 patients had higher body mass index (BMI) and Non-alcoholic fatty liver disease (NAFLD) co-morbidity rates. According to the study, NAFLD patients had a higher risk of severe COVID-19 disease development and a longer period of viral shedding [57]. Patients younger than 60 years old with NAFLD and SARS-CoV-2 infection have a higher risk of developing the severe disease [58]. NAFLD patients with greater non-invasive liver fibrosis scores showed a higher likelihood of developing severe COVID-19 illness, notwithstanding metabolic comorbidities [59].

\section{Diabetes}

Based on Abu-Farha et al.'s (2020) study, diabetes affects 26.8\% of older COVID-19 patients at a higher risk of death [60]. COVID19-positive hyperglycemic patients had a greater cumulative incidence of severe illness than normoglycemic controls, according to previous research [61, 62]. One probable component that may play a role in elevating the risk in persons with diabetes is a defective innate and adaptive immune response, which is characterized by a state of chronic and low-grade inflammation that can lead to rapid systemic metabolic alterations. Compared to patients under metabolic control, SARS-CoV-2 patients who had previously been diagnosed with diabetes or hyperglycemia had greater mortality and morbidity rates. During the SARS-CoV-2 outbreak in Italy, patients with diabetes had a higher prevalence, severity of disease, and mortality. Moreover, they also had a greater rate of ICU admission. Diabetes is also linked to a higher risk of severe to critical disease in 14 to 32 percent of COVID-19 patients [63]. Other studies found that those diagnosed with diabetes or other comorbidities were more likely to be admitted to the ICU than nonICU patients. The percentage of ICU admission for COVID-19 patients with diabetes or other comorbidities was 22.2 percent but 5.9 percent only for COVID-19 patients without diabetes or other comorbidities [64]. Several factors have been linked to the risk and severity of SARS-CoV2 infection in diabetics, including increased expression of ACE2, furin, and IL-6, as well as reduced T-cell activity [65].

\section{Dementia}

In individuals with dementia, such as vascular dementia and Alzheimer's disease, the blood-brain barrier (BBB) is broken, enabling bacteria and viruses greater access to the brain and rendering them more susceptible to bacterial, viral, and fungal infection. Moreover, Dementia-related cognitive impairment may make it difficult for the patient to adhere to COVID-19 preventative techniques such as social distancing, mask-wearing, and frequent hand cleaning. As a result, persons with dementia are more likely to get COVID-19.

According to Wang et al. (2021) study, it has been found that patients with dementia had a higher risk of COVID-19 than those without dementia (adjusted odds ratio (AOR): 2.00); with vascular dementia having the strongest effect 
(AOR: 3.17); presenile dementia (AOR: 2.62); and Alzheimer's disease (AOR: 1.86), senile dementia (AOR: 1.99) and post-traumatic dementia (AOR: 1.67). The results show a significant value $(p<0.001)$ [66]. In patients with dementia and COVID-19, the 6-month death and hospitalization risks were 20.99 percent and 59.26 percent, respectively. According to a survey of patients at an Italian memory center, up to $31 \%$ of dementia patients had severe cognitive decline during the first month of confinement, with 54\% experiencing increased agitation, apathy, and depression [67].

\section{Long COVID-19 in young children}

According to the findings of the first research of protracted COVID-19 in children, half of the children aged 6 to 16 who get the virus have at least one symptom lasting longer than four months, with 42.6 percent of these symptoms interfering with everyday activities. These preliminary findings are based on periodic assessments of 129 Italian infants diagnosed with COVID-19 at the Gemelli University Hospital in Rome between March and November 2020 [68].

According to the latest study from the United Kingdom (UK) Office for National Statistics, 12.9 percent of UK youngsters aged between 2 and 11 and 14.5 percent of children aged between 12 and 16 still show symptoms five weeks after their initial infection of SARS-CoV-2. Since March 2020, about 500,000 young children in the UK have suffered from COVID-19 positive. Most medical organizations claim that recovering from COVID-19 usually takes a few days or weeks and that most people will be fully recovered by 12 weeks [69].

After contracting COVID-19 in March 2020, a 14-year-old girl in the UK became vacant, feeble, and unresponsive. After three weeks in bed, she conducted some gentle activity in the garden and gripped her chest, complaining of heart trouble. She could not walk back to her bed. She stayed in bed for 7 months [68].

A family with five children in the UK has long COVID-19 after contracting the virus early in the pandemic (March 2020). The 4-year-old still suffers a stiff neck, lethargy, gastrointestinal difficulties, and headaches almost a year after contracting COVID-19. The 10-year-old is tired and has stomach issues and pain around his heart [68].

One family with five children aged 9-15 years suffered from COVID-19 in early March 2020. Four of the five children had no comorbidities.
The fifth child is affected by asthma and allergies. $\mathrm{He} / \mathrm{She}$ is also autistic. Despite the fact that none of the children had been taken to the hospital when COVID-19 was detected, the girl with pre-existing comorbidities was admitted after 90 days of COVID-19 diagnosis. She was admitted due to a peri-myocarditis. Furthermore, many people with long-term COVID-19 had a modest ailment at the start. All five children suffered from fatigue, dyspnoea, joint stiffness, and chest discomfort. Other symptoms included neurological issues, skin rashes, sleep issues, sadness, physical discomfort, and remitting fever [70].

A recent study on UK school-aged children (512 years old) who contracted with SARS-CoV-2 infection was conducted. The data was collected between $24^{\text {th }}$ March 2020 and $22^{\text {nd }}$ February 2021. 25 of 588 young children suffered from fatigue, headache and anosmia for the last 58 days after contracted with the virus [71].

Based on the most current Australian study (April 2021), 151 young children aged 3 years were monitored for three-six months. The majority of the children had a moderate or asymptomatic illness, with only $8 \%$ having persistent symptoms. However, other modest long-term outcome studies in children reveal that more than half of them have at least one persistent symptom after four months of contracting the virus [72].

More research is needed to comprehend better the occurrence and prognosis of long COVID-19 in young children. Affected children require appropriate follow-up visits that take into account child-specific characteristics, and the approach could be of assistance

\section{Conclusion}

COVID-19 has impacted people with noncommunicable diseases and young children. According to the evidence, in this study, chronic lung, kidney and liver diseases, diabetes mellitus, cancer and dementia appear to be major risk factors for disease caused by coronavirus infections like COVID-19. Even though there is no medication permitted until now, scientists are still researching new anti-viral drugs with less or no side effects to cure the infection. Worldwide, Pfizer, AstraZeneca, Sinovac, Moderna, etc. vaccines are administered successfully to people. However, there are some limitations to these vaccines. For example, young children are not allowed to give these vaccines. Every individual's support by 
obeying the government's law is required to eradicate this pandemic soon. Researchers focus more on severe sickness and mortality in adult and older people until far. However, there is urgent to investigate the COVID-19 pandemic's effects on all young children. This includes young children who have had acute infection followed by longCOVID. This type of research will aid in the development of clinical features, mechanisms, pathways, and plans of action for preventing negative outcomes.

\section{Acknowledgement}

We would like to thank front liners who are fighting against this deadly COVID-19 pandemic.

\section{References}

1. Singhal T (2020) A review of coronavirus disease-2019 (COVID-19). The Indian Journal of Pediatrics 87(4): 281-286. doi: 10.1007/s12098-020-03263-6

2. Elengoe A (2020) COVID-19 outbreak in Malaysia. Osong Public Health and Research Perspectives 11(3): 93.

3. Mahase E (2021) COVID-19: What new variants are emerging and how are they being investigated? British Medical Journal 372: n158.

4. Lopez Bernal J, Andrews N, Gower C et al. (2021) Effectiveness of COVID-19 vaccines against the B. 1.617. 2 (Delta) variant. New England Journal of Medicine 385: 585-594.

5. Darby AC, Hiscox JA (2021) COVID-19: variants and vaccination. British Medical Journal 372: n771.

6. Guo YR, Cao QD, Hong ZS et al. (2020) The origin, transmission and clinical therapies on coronavirus disease 2019 (COVID-19) outbreak-an update on the status. Military Medical Research 7(1): 1-10.

7. Forni G, Mantovani A (2021) COVID-19 vaccines: where we stand and challenges ahead. Cell Death \& Differentiation 28(2): 626-639.

8. World Health Organization. COVID-19 vaccine tracker and landscape. https://www.who.int/publications/m/item/draft-landscape-of-COVID-19-candidatevaccines. Accessed: 24 August, 2021

9. World Health Organization. Coronavirus disease (COVID-19): Vaccines. https://www.who.int/newsroom/q-a-detail/coronavirus-disease-(COVID-19)-vaccines Accessed: 28 October, 2020.

10. COVID-19 Coronavirus pandemic. Worldometer.https://www.worldometers.info/coronavirus/. Accessed: 24 August, 2021.

11. Onder G, Rezza G, Brusaferro S (2020) Case-fatality rate and characteristics of patients dying in relation to COVID-19 in Italy. The Journal of the American Medical Association 323(18): 1775-1776.

12. Zhang JJ, Dong X, Cao YY et al. (2020) Clinical characteristics of 140 patients infected with SARS-CoV-2 in Wuhan, China. Allergy 75(7): 1730-1741.

13. Huang C, Wang Y, Li X et al. (2020) Clinical features of patients infected with 2019 novel coronavirus in Wuhan, China. Lancet 395(10223): 497-506.
14. Wang D, Hu B, Hu C et al. (2020) Clinical characteristics of 138 hospitalized patients with 2019 novel coronavirus-infected pneumonia in Wuhan, China. The Journal of the American Medical Association 323(11): 10611069.

15. Bhatraju PK, Ghassemieh BJ, Nichols M et al. (2020) COVID-19 in critically Ill patients in the Seattle region case series. New England Journal of Medicine 382(21): 2012-2022.

16. Guo T, Fan Y, Chen M et al. (2020) Cardiovascular implications of fatal outcomes of patients with coronavirus disease 2019 (COVID-19). The Journal of the American Medical Association Cardiology 5(7): 811-818.

17. Guan WJ, Ni ZY, Hu Y et al. (2020) Clinical characteristics of coronavirus disease 2019 in China. New England Journal of Medicine 382: 1708-1720.

18. Lian J, Jin X, Hao S et al. (2020) Analysis of epidemiological and clinical features in older patients with Corona Virus Disease 2019 (COVID-19) out of Wuhan. Clinical Infectious Disease 2020:1-8.

19. Guan WJ, Liang WH, Zhao Y et al. (2020) Comorbidity and its impact on 1590 patients with COVID-19 in China: A nationwide analysis. European Respiratory Journal 55: 2000547.

20. Liang W, Guan W, Chen R et al. (2020) Cancer patients in SARS-CoV-2 infection: A nationwide analysis in China. Lancet Oncology 21: 335-337.

21. Li X, Xu S, Yu M et al. (2020) Risk factors for severity and mortality in adult COVID-19 inpatients in Wuhan. The Journal of Allergy and Clinical Immunology 146: 110-118.

22. Zhang L, Zhu F, Xie L et al. (2020) Clinical characteristics of COVID-19-infected cancer patients: A retrospective case study in three hospitals within Wuhan, China. Annals of Oncology 31: 894-901.

23. Rentsch CT, Kidwai-Khan F, Tate JP et al. (2020) Patterns of COVID-19 testing and mortality by race and ethnicity among United States veterans: A nationwide cohort study. PLoS Medicine 17(9): e1003379.

24. Praveen S, Ashish K, Anikhindi SA et al. (2020) Effect of COVID-19 on pre-existing liver disease: What Hepatologist should know? Journal of Clinical and Experimental Hepatology 11(4): 484-493.

25. Phipps MM, Barraza LH, LaSota ED, Sobieszczyk ME, Pereira MR, Zheng EX, Fox AN, Zucker J, Verna EC (2020) Acute liver injury in COVID-19: prevalence and association with clinical outcomes in a large US cohort. Hepatology 72(3): 807-817.

26. Reyes-Bueno JA, Mena-Vázquez N, Ojea-Ortega T et al. (2020) Case fatality of COVID-19 in patients with neurodegenerative dementia. Neurología 35(9): 639-645.

27. Guan W, Ni Z, Hu Y et al. (2020) Clinical characteristics of coronavirus disease 2019 in China. New England Journal of Medicine 382(18): 1708-1720.

28. Hotchin NA, Read R, Smith D, Crawford, DH (1989). Active Epstein-Barr virus infection in post-viral fatigue syndrome. Journal of Infection 18(2): 143-150.

29. Gaiha SM, Cheng J, Halpern-Felsher B (2020) Association between youth smoking, electronic cigarette use, and COVID-19. Journal of Adolescent Health 67(4): 519-523.

30. Sohrabi C, Alsafi Z, O’Neill N et al. (2020) World Health Organization declares global emergency: A 
review of the 2019 novel coronavirus (COVID-19). International Journal of Surgery, 76, 71-76.

31. Ost DE (2020). Bronchoscopy in the Age of COVID-19. Journal of Bronchology \& Interventional Pulmonology 27(3): 160-162.

32. Cheung JCH, Ho LT, Cheng JV et al. (2020) Staff safety during emergency airway management for COVID-19 in Hong Kong. The Lancet Respiratory Medicine 8(4): e19.

33. Spagnolo P, Balestro E, Aliberti S et al. (2020) Pulmonary fibrosis secondary to COVID-19: a call to arms? The Lancet Respiratory Medicine 8 (8): 750-752.

34. Lee SC, Son KJ, Han CH et al. (2021) Impact of COPD on COVID-19 prognosis: A nationwide populationbased study in South Korea. Scientific Reports 11(1): 18.

35. Bilaçeroğlu S. Letter from Turkey: Impact of COVID-19 on respiratory diseases (2021) Respirology.

36. Han Y, Saran R (2020) Global Dialysis Perspective: United States. Kidney360.

37. Hosen MS, Nafiujjaman M, Nishat TR (2021) Alleviating the Financial Burden for Dialysis Patients in Lowand Middle-Income Countries during the COVID-19 Pandemic. International Journal of Health Life Science, 7(1), e106682.

38. Kato S, Chmielewski M, Honda $\mathrm{H}$ et al. (2008) Aspects of immune dysfunction in end-stage renal disease. Clinical Journal of the American Society of Nephrology 3(5): 1526-1533.

39. Fang L, Karakiulakis G, Roth M (2020) Are patients with hypertension and diabetes mellitus at increased risk for COVID-19 infection? The Lancet Respiratory Medicine 8(4): e21.

40. O'Grady NP, Alexander M, Dellinger EP et al. (2002) Guidelines for the prevention of intravascular catheterrelated infections. Centers for Disease Control and Prevention. MMWR. Recommendations and Reports: Morbidity and Mortality Weekly Report. Recommendations and Reports 51(RR-10): 1-29.

41. Chang AY, Cullen MR, Harrington RA, Barry M (2021) The impact of novel coronavirus COVID-19 on noncommunicable disease patients and health systems: a review. Journal of Internal Medicine, 289(4): 450-462.

42. Saran R, Robinson B, Abbott KC et al. (2020) US renal data system 2019 annual data report: epidemiology of kidney disease in the United States.

43. ERA-EDTA Council, ERACODA Working Group (2021) Chronic kidney disease is a key risk factor for severe COVID-19: a call to action by the ERA-EDTA. Nephrology Dialysis Transplantation 36: 87-94.

44. Hsu CM, Weiner DE, Aweh G (2021) COVID-19 Infection among US dialysis patients: risk factors and outcomes from a national dialysis provider. American Journal of Kidney Diseases 77(5): 748-756.

45. Prevention C. for D. C. and. (n.d.). People who are at increased risk for severe illness. Accessed: 29 April, 2020.

46. Liang W, Guan W, Chen R et al. (2020) Cancer patients in SARS-CoV-2 infection: a nationwide analysis in China. The Lancet Oncology 21(3): 335-337.

47. Xia Y, Jin R, Zhao J et al. (2020) Risk of COVID-19 for cancer patients. Lancet Oncology: 21(4).

48. Connors JM, Levy JH (2020) COVID-19 and its implications for thrombosis and anticoagulation. The Journal of the American Society of Hematology, 135(23): 20332040.
49. Runowicz CD, Leach CR, Henry NL et al. (2016) American cancer society/American society of clinical oncology breast cancer survivorship care guideline. CA: A Cancer Journal for Clinicians 66(1): 43-73.

50. Remuzzi A, Remuzzi G (2020) COVID 19 and Italy: what next? Lancet 395 (10231): P1225-1228.

51. Liang W, Guan W, Chen R (2020) Cancer patients in SARS-CoV-2infection: a nationwide analysis in China. Lancet Oncology 21(3): 335-337.

52. Pan L, Mu M, Yang P et al. (2020) Clinical characteristics of COVID-19 patients with digestive symptoms in Hubei, China: a descriptive, cross-sectional, multicenter study. The American Journal of Gastroenterology: 115.

53. Gu J, Han B, Wang J (2020) COVID-19: gastrointestinal manifestations and potential fecal-oral transmission. Gastroenterology 158(6): 1518-1519.

54. Kumar D, Tellier R, Draker R et al. (2003) Severe Acute Respiratory Syndrome (SARS) in a liver transplant recipient and guidelines for donor SARS screening. American Journal of Transplantation 3(8): 977-981.

55. Chopra V, Toner E, Waldhorn R, Washer L (2020) How should US hospitals prepare for coronavirus disease 2019 (COVID-19)? American College of Physicians 172(9): 621-622.

56. Wang Q, Davis PB, Xu R (2021) COVID-19 risk, disparities and outcomes in patients with chronic liver disease in the United States. EClinical Medicine 31:100688.

57. Ji D, Qin E, Xu J et al. (2020) Non-alcoholic fatty liver diseases in patients with COVID-19: A retrospective study. Journal of Hepatology 73: 451-453.

58. Zhou YJ, Zheng KI, Wang XB et al. (2020) Younger patients with MAFLD are at increased risk of severe COVID-19 illness: A multicenter preliminary analysis. Journal of Hepatology 73: 719-721.

59. Targher G, Mantovani A, Byrne CD et al. (2020) Risk of severe illness from COVID-19 in patients with metabolic dysfunction-associated fatty liver disease and increased fibrosis scores. Gut 69: 1545-1547.

60. Abu-Farha M, Al-Mulla F, Thanaraj TA et al. (2020) Impact of diabetes in patients diagnosed with COVID-19. Frontiers in Immunology 11: 3112.

61. Sardu C, D'onofrio N, Balestrieri ML et al. (2020) Outcomes in patients with hyperglycemia affected by COVID-19: Can we do more on glycemic control? Diabetes Care 43 (7):1408-1415.

62. Sardu C, D'onofrio N, Balestrieri ML et al. (2020) Hyperglycaemia on admission to hospital and COVID-19. Diabetologia 63(11): 2486-2487.

63. Zhang JJ, Dong X, Cao YY et al. (2020) Clinical characteristics of 140 patients infected with SARS-CoV-2 in Wuhan, China. Allergy 75 (7): 1730-1741.

64. Wang D, Hu B, Hu C et al. (2020) Clinical characteristics of 138 hospitalized patients with 2019 novel coronavirus-infected pneumonia in Wuhan, China. The Journal of the American Medical Association 323(11): 10611069.

65. Feng SQ, Chen XD, Xia T et al. (2006) Cloning, chromosome mapping and expression characteristics of porcine ANGPTL3 and -4. Cytogenet Genome Res 114(1): 44-49.

66. Wang Q, Davis PB, Gurney ME, Xu R (2021) COVID19 and dementia: Analyses of risk, disparity, and outcomes from electronic health records in the US. Alzheimer's \& Dementia 17(8): 1297-1306. 
67. Hanratty B, Burton JK, Goodman C et al. (2020) COVID-19 and lack of linked datasets for care homes. British Medicine Journal 369: m2463.

68. News Coronavirus. Long-term effects children with long COVID. https://www.ncbi.nlm.nih.gov/pmc/articles/PMC7927578/pdf/main.pdf. Accessed on $23 \mathrm{Au}-$ gust, 2021.

69. Riphagen S, Gomez X, Gonzalez-Martinez C et al. (2020) Hyperinflammatory shock in children during COVID-19 pandemic. The Lancet 395(10237): 16071608.
70. Carfì A, Bernabei R, Landi F (2020) Persistent symptoms in patients after acute COVID-19. The Journal of the American Medical Association 324(6): 603-605.

71. Molteni E, Sudre CH, Canas LS et al. (2021) Illness duration and symptom profile in a large cohort of symptomatic UK school-aged children tested for SARS-CoV-2. medRxiv.

72. Say D, Crawford N, McNab S et al. (2021) Post-acute COVID-19 outcomes in children with mild and asymptomatic disease. The Lancet Child \& Adolescent Health 5(6):e22-3. 
This page is intentionally left blank. 